\title{
Gene hunting of the Genetic Analysis Workshop 16 rheumatoid arthritis data using rough set theory
}

\author{
Chatchawit Aporntewan ${ }^{1,2}$, David H Ballard ${ }^{3}$, Ji Young Lee ${ }^{4}$, Joon Sang Lee ${ }^{5}$, \\ Zheyang $\mathrm{Wu}^{5}$ and Hongyu Zhao ${ }^{* 5,6}$
}

Addresses: ${ }^{1}$ Department of Psychiatry, Yale University, 300 George Street, Suite 503, New Haven, Connecticut 06511, USA, ${ }^{2}$ Department of Mathematics, Chulalongkorn University, 254 Phayathai Road, Pathumwan, Bangkok, 10330, Thailand, ${ }^{3}$ Program in Computational Biology and Bioinformatics, Yale University, 100 Howe Street, New Haven, Connecticut 06511, USA, ${ }^{4}$ Biostatistics Resource, Keck Laboratory, Yale University, 300 George Street, Suite 503, New Haven, Connecticut 06511, USA, ${ }^{5}$ Department of Epidemiology and Public Health, Yale University, 300 George Street, Suite 503, New Haven, Connecticut 06511, USA and ${ }^{6}$ Department of Genetics, Yale University, 300 George Street, Suite 503, New Haven, Connecticut 06511, USA

E-mail: Chatchawit Aporntewan - chatchawit.aporntewan@yale.edu; David H Ballard - david.ballard@yale.edu;

Ji Young Lee - jiyoung.lee@yale.edu; Joon Sang Lee - joonsang.lee@yale.edu; Zheyang Wu - zheyang.wu@yale.edu;

Hongyu Zhao* - hongyu.zhao@yale.edu

${ }^{*}$ Corresponding author

from Genetic Analysis Workshop 16

St Louis, MO, USA 17-20 September 2009

Published: 15 December 2009

BMC Proceedings 2009, 3(Suppl 7):SI26 doi: 10.1 I86/1753-656I-3-S7-SI26

This article is available from: http://www.biomedcentral.com/I753-656I/3/S7/SI26

(C) 2009 Aporntewan et al; licensee BioMed Central Ltd.

This is an open access article distributed under the terms of the Creative Commons Attribution License (http://creativecommons.org/licenses/by/2.0), which permits unrestricted use, distribution, and reproduction in any medium, provided the original work is properly cited.

\begin{abstract}
We propose to use the rough set theory to identify genes affecting rheumatoid arthritis risk from the data collected by the North American Rheumatoid Arthritis Consortium. For each gene, we employ generalized dynamic reducts in the rough set theory to select a subset of single-nucleotide polymorphisms (SNPs) to represent the genetic information from this gene. We then group the study subjects into different clusters based on their genotype similarity at the selected markers. Statistical association between disease status and cluster membership is then studied to identify genes associated with rheumatoid arthritis. Based on our proposed approach, we are able to identify a number of statistically significant genes associated with rheumatoid arthritis. Aside from genes on chromosome 6, our identified genes include known disease-associated genes such as PTPN22 and TRAFI. In addition, our list contains other biologically plausible genes, such as ADAMI5 and AGPAT2. Our findings suggest that ADAMI5 and AGPAT2 may contribute to a genetic predisposition through abnormal angiogenesis and adipose tissue.
\end{abstract}

\section{Introduction}

Rheumatoid arthritis (RA) is a chronic and systemic autoimmune disorder. It is characterized by synovitis an inflammation of synovial membranes, which enclose joint. The afflicted joints become warm, swollen, tender, stiff, and in the final stage, deformed. RA is believed to be a heterogeneous disease because the manifestations are greatly varied across patients in terms of severity, 
progression, and response to therapy. It is estimated that genetic factors account for $60 \%$ of disease susceptibility [1]. The association between human leukocyte antigen (HLA) genes and RA has been well established, although HLA genes only account for $30 \%$ of the genetic contribution [1]. This distribution suggests that a genetic predisposition to RA involves non-HLA genes. There are many ongoing efforts to identify non-HLA genes associated with RA. Recent studies indicate that PTPN22, OLIG3/TNFAIP3, STAT4, and TRAF1/C5 genes may be involved in RA [2-5], and it is believed that many other genes are yet to be discovered.

To incorporate prior genome annotation information, we consider a gene-based analysis in this manuscript with the hope that a joint analysis of all the markers, e.g., single-nucleotide polymorphisms (SNPs), in a gene may increase statistical power for gene detection [6]. To achieve this goal, we employ the rough set theory (RST) [7], which is a method for feature selection to select informative SNPs for association analysis. Although a previous study concluded that RST did not identify disease-related loci [8], it only considered relative reducts, one of many options (e.g., global reducts, dynamic reducts, and generalized dynamic reducts) in RST for feature selection. The more robust definitions, such as generalized dynamic reducts, may offer higher power to detect genes associated with disease. We combined SNPs selected from RST to define joint patterns across these SNPs. We then clustered the joint patterns so that subjects sharing similar genotypes are grouped together. Statistical association was studied between disease status and cluster membership. In the following, we detail these steps and report findings from the analysis of North American Rheumatoid Arthritis Consortium (NARAC) data.

\section{Methods}

The genome-wide RA data was collected by the NARAC and provided by the Genetic Analysis Workshop 16. SNPs with HWE $p$-value $<0.001$ or MAF $<0.01$ or with a missing percentage of an SNP or an individual $>0.1$ were excluded. Subsequently, 500,884 SNPs from 1,140 controls and 862 cases remained. The remaining SNPs were assigned to their corresponding genes according to the gene annotation attached to the data. Note that the gene annotation assigned every SNP to a gene. The SNPs that were not in any gene regions were assigned to neighboring genes. In addition to case-control status, anti-cyclic citrullinated peptide (anti-CCP) and rheumatoid factor (RF) were used to classify clinical subgroups of RA patients. The subjects whose RF $>40 \mathrm{IU} / \mathrm{ml}$ were classified as RF+. A total of 1,961 subjects had anti-CCP data (1,330 anti-CCP+), and a total of 861 subjects had RF data (759 RF+).
We first give an overview of the RST [7]. Let $A=(U, A \cup$ $\{d\})$ denote a decision table in which $U$ is a set of subjects, $A$ is a full set of SNPs in a specific gene, and $d$ is a decision variable (case/control). An example of a decision table is shown in Table 1. A reduct is a minimal set of SNPs that are relevant to the decision variable. $B \subset$ $A$ is a relative reduct if $B$ is a minimal set such that $\partial_{B}=\partial_{A}$, where $\partial_{B}$ denotes a decision based on $B$. In Table 1 , the universe $U$ is $\{$ Subject $1, \ldots$, Subject6 $\}$ and the decision $V_{d}$ is $\{$ Case, Control $\}$. The function $\partial_{B} U \rightarrow V_{d}$ is defined

as $\partial \downarrow B(x)=\{v \in V \downarrow d$ : $x$ might be $v$ if we can observe only SNPs of $x$ in $B\}$. For example,

$$
\begin{aligned}
& \partial_{\{\text {SNP1,SNP } 2\}}(\text { Subject } 1)=\{\text { Case, } \text { Control }\} \\
& \partial_{\{\text {SNP1,SNP2 }\}}(\text { Subject } 4)=\{\text { Case }, \text { Control }\} \\
& \partial_{\{\text {SNP1,SNP2 }\}}(\text { Subject } 6)=\{\text { Case, } \text { Control }\},
\end{aligned}
$$

whereas

$$
\partial_{\{\mathrm{SNP} 1, \mathrm{SNP} 2\}}(\text { Subject3) }=\{\text { Case, } \text { Control }\} .
$$

Table 1 yields two relative reducts, $\{$ SNP1, SNP3 $\}$ and $\{\mathrm{SNP} 2, \mathrm{SNP} 3\}$. The set of all relative reducts of decision table $A$ is denoted by $\operatorname{RED}(\mathrm{A}, d)$. Note that the above definition of relative reducts is also valid for a decision table with missing values.

The relative reducts are very sensitive to a small change in the decision table. Similar to the case in which a small prediction error is achieved without cross-validation, the prediction is not reliable because it overfits the data. Dynamic reducts provide a more robust solution to feature selection. It is defined as follows:

$$
D R(\mathbf{A}, \mathbf{F})=R E D(\mathbf{A}, d) \bigcap \bigcap_{\mathrm{B} \in \mathrm{F}} R E D(\mathbf{B}, d)
$$

$D R(\mathbf{A}, \mathbf{F})$ denotes the set of all dynamic reducts of decision table $\mathbf{A}$, and $\mathbf{F}$ denotes a finite set of subtables

Table I: An example of a decision table

\begin{tabular}{lcccc}
\hline Subject $^{\text {a }}$ & SNPI & SNP2 & SNP3 & Status \\
\hline 1 & 1 & 2 & 2 & Case \\
2 & 2 & 1 & 2 & Case \\
3 & 2 & 2 & 3 & Case \\
4 & 1 & 2 & 1 & Control \\
5 & 2 & 1 & 2 & Control \\
6 & 1 & 2 & 3 & Case \\
\hline
\end{tabular}

${ }^{a}$ The decision table contains all subjects in a study and all SNPs in a specific gene. SNP $\in\{I, 2,3$, missing $\}$, where $I=a a, 2=a A, 3=A A$, Status $\in\{$ Case, Control\}. This table was adapted from that in Pawlak [24]. 
based on a subset of all the rows in the decision table. The default sizes of the subtables are $50 \%, 60 \%, 70 \%$, $80 \%$, and $90 \%$ [9]. For each size, ten subtables are randomly constructed. In each subtable, the ratio of cases to controls is approximately equal to that of the full table. A more relaxed definition is generalized dynamic reducts, denoted by $\operatorname{GDR}(\mathbf{A}, \mathbf{F})$, where

$$
\operatorname{GDR}(\mathbf{A}, \mathbf{F})=\bigcap_{\mathrm{B} \in \mathrm{F}} R E D(\mathbf{B}, d) .
$$

The definition of GDR in Eq. (6) can be further loosened by equipping it with an epsilon,

$$
\operatorname{GDR}_{\in}(\mathrm{A}, \mathrm{F})=\{C \subseteq A \text { : stability coefficient of } C>1-\in\},
$$

where the stability coefficient (SC) is

$$
\mathrm{SC}=\frac{\operatorname{card}(\{\mathrm{B} \in \mathrm{F}: C \in R E D(\mathrm{~B}, d)\})}{\operatorname{card}(\mathrm{F})} .
$$

card(S)denotes the cardinality of set $\mathrm{S}$. The value of SC indicates the uniformity of a generalized dynamic reduct in the full decision table.

We set $\varepsilon$ at 0.5 to discard the reducts that are statistically significant, but not stable (small SC). Among all the reducts found, we chose the one having the highest SC as a set of representative SNPs for a gene. The computational burden can be high when a gene contains hundreds of SNPs. Our empirical study showed that RST often selects among the top-ten SNPs ranked by an allelic test. To relieve the computational burden, for every gene we pre-selected 10 SNPs through allelic tests. There are 8,105 genes $(49.01 \%)$ that contain more than 10 SNPs.

Starting from the top ten or fewer SNPs, RST yields a set of selected SNPs and their joint genotypes form a joint genotype pattern. We then applied the Pearson's chisquare test to calculate the $p$-value for genetic association based on this $n \times 2$ table, where $n$ is the number of distinct patterns, and the two columns correspond to cases and controls. The patterns with at least one missing SNP are excluded from the statistical test (no imputation). We use permutations to correct for multiple comparisons. For each permutation, the case and control status is shuffled, and the reducts are recomputed. We conducted 1,000 permutations to derive the empirical statistical significance level.

We used the complete-link clustering and agglomerative algorithm [10] to group individuals based on their joint genotype patterns. The optimal number of clusters was determined by the Dunn index [11]. The clustering algorithm was terminated when

$$
a(k, P)<1,
$$

where

$$
a(k, P)=\left(1 \leq \boldsymbol{q} \leq \boldsymbol{k}^{\mathrm{min}} \frac{\min _{1 \leq \boldsymbol{r} \boldsymbol{r}} k \operatorname{dist}\left(X_{\boldsymbol{q}}, X_{r}\right)}{\left(1 \leq p \leq k^{\max }\right)} \operatorname{diam}\left(X_{p}\right),\right.
$$

$$
\begin{gathered}
\operatorname{diam}(A) \triangleq\left(x, y \in A^{\max } d(x, y),\right. \\
\operatorname{dist}(A, B) \triangleq x \in \min _{y \in B}^{A^{d(x, y)},}
\end{gathered}
$$

$$
A, B \subset X \text { and } A, B \neq \phi,
$$

where $k$ denotes the number of clusters, $P=\left\{X_{1}, \ldots, X_{k}\right\}$ denotes a $k$-partition of $X$, and $X$ denotes the complete set of all items that we want to cluster. For instance, $X=$ $\{\boldsymbol{a}, \boldsymbol{b}, \boldsymbol{c}, \boldsymbol{d}, \boldsymbol{e}, f\}, \boldsymbol{k}=3$ and $\boldsymbol{P}=\{\{\boldsymbol{a}, \boldsymbol{b}, \boldsymbol{c}\},\{d\},\{\boldsymbol{e}, f\}\}$ The diameter of cluster $A$ is $\operatorname{diam}(A)$, and the distance between clusters $A$ and $B$ is $\operatorname{dist}(\boldsymbol{A}, \boldsymbol{B})$. The distance between items $x$ and $y, d(x, y)$, is measured by mutual information (MI) [12]. MI clustering is motivated by the observation that subjects who shared similar haplotypes were put in the same cluster [13]. Another distance measure proposed in the literature is allele sharing (AS), which was used to classify HapMap populations [14]. We apply both MI and AS clustering, and then calculate the statistics of clustered patterns.

\section{Results}

Based on the approach discussed above, we have identified non-HLA genes associated with RA (Table 2). These genes include some known genes, TRAF1 and PTPN22, and also include novel genes. In the following, we focus our discussion on two genes that are more biologically plausible, namely, ADAM15 and AGPAT2. Because the high degrees of freedom for these tables, we reduce the total number of patterns considered through clustering. The results are also shown in Table 2. MI clustering altered the ranks of PTPN22, AGPAT2, ADAM15, TRAF1 to three, four, five, and six, respectively, whereas the ranks altered by AS clustering were five, nine, seven, and six.

Because this data set is enriched for many gene signals on chromosome 6 , all genes on chromosome 6 are excluded from Table 2, and they are shown separately in Table 3. Among HLA genes, HLA-DRB1 shows the strongest association to RA. BTNL2 seems to be the best candidate 
Table 2: Top-ranked genes associated with RA (22 autosomes, except chromosome 6)

\begin{tabular}{|c|c|c|c|c|c|c|c|c|c|}
\hline \multirow[t]{2}{*}{ Chr. } & \multirow[t]{2}{*}{ Gene symbol $^{\mathrm{a}}$} & \multirow[t]{2}{*}{ No. SNPs ${ }^{b}$} & \multirow[t]{2}{*}{$S^{c}$} & \multicolumn{2}{|c|}{ No clustering ${ }^{d}$} & \multicolumn{2}{|c|}{ MI clustering } & \multicolumn{2}{|c|}{ AS clustering } \\
\hline & & & & $p$-value & $d f^{e}$ & $p$-value ${ }^{f}$ & $d f^{e}$ & $p$-value ${ }^{f}$ & $d f^{e}$ \\
\hline 16 & Cl6orf57 & $2 / 2$ & 1 & $6.06 \times 10^{-8}$ & 5 & $6.06 \times 10^{-8}(1)$ & 5 & $1.65 \times 10^{-2}(10)$ & 3 \\
\hline 21 & KRTAPI0-6 & $2 / 2$ & 1 & $8.06 \times 10^{-6}$ & 6 & $8.06 \times 10^{-6}(2)$ & 6 & $4.42 \times 10^{-7}(\mathrm{I})$ & 3 \\
\hline 19 & DEDD2 & $3 / 3$ & 1 & $3.29 \times 10^{-5}$ & 12 & $3.35 \times 10^{-3}(9)$ & 2 & $1.84 \times 10^{-6}(2)$ & 6 \\
\hline 9 & AGPAT2 & $4 / 4$ & I & $4.57 \times 10^{-5}$ & 30 & $4.57 \times 10^{-5}(4)$ & 5 & $3.30 \times 10^{-3}(9)$ & 18 \\
\hline 9 & TRAFI & $3 / 3$ & $\mathbf{I}$ & $7.35 \times 10^{-5}$ & II & $5.90 \times 10^{-5}(6)$ & 2 & $8.91 \times 10^{-6}(6)$ & 6 \\
\hline 15 & ODF3LI & $4 / 4$ & I & $1.00 \times 10^{-4}$ & 29 & $1.00 \times 10^{-4}(7)$ & 29 & $1.89 \times 10^{-6}(3)$ & 17 \\
\hline 19 & RSHLI & $3 / 3$ & 1 & $1.02 \times 10^{-4}$ & 12 & $1.53 \times 10^{-3}(8)$ & 1 & $1.09 \times 10^{-3}(8)$ & 6 \\
\hline 11 & CDC42BPG & $3 / 3$ & I & $1.04 \times 10^{-4}$ & 12 & $7.95 \times 10^{-1}(10)$ & 1 & $2.55 \times 10^{-6}(4)$ & 6 \\
\hline 1 & ADAM I 5 & $7 / 7$ & 1 & $1.08 \times 10^{-4}$ & 64 & $5.62 \times 10^{-5}(5)$ & 49 & $2.05 \times 10^{-5}(7)$ & 40 \\
\hline I & PTPN22 & $6 / 7$ & 0.5 & $1.24 \times 10^{-4}$ & 34 & $1.52 \times 10^{-5}(3)$ & 27 & $3.58 \times 10^{-6}(5)$ & 20 \\
\hline
\end{tabular}

${ }^{a}$ The genes are sorted by $p$-value (no clustering). Only the top ten genes in this table were clustered.

${ }^{\mathrm{b}}$ The number of selected SNPs and the total number of SNPs that were genotyped.

'SC, stability coefficient.

${ }^{\mathrm{d}}$ The adjusted $p$-values (no clustering) for all genes in the table are less than $0.00 \mathrm{I}$ ( 1,000 permutations).

e df, degree of freedom, which is the number of clusters subtracted by one.

${ }^{\mathrm{f}}$ The numbers in parenthesis are the ranks according to $\mathrm{MI}$ and AS clustering.

${ }^{g}$ Bold font indicates the genes that have been reported in the literature (PTPN22 and TRAFI) and the genes that we have found that are biologically plausible (ADAMI5 and AGPAT2).

for non-HLA genes on this chromosome but the SC is relatively small, which implies that BTNL2 may only affect a subset of all RA cases.

To determine the RA subgroups associated with BTNL2 $(\mathrm{SC}=0.36)$ and PTPN22 (SC $=0.54)$, we conducted association studies in two clinical subgroups classified by anti-CCP and RF (Table 4). Our analysis shows that BTNL2 was strongly associated with anti-CCP, but BTNL2 was not associated with RF (Table 4). BTNL2 is close to HLA genes, which are associated with the production of anti-CCP [15]. As a result, the association of BTNL2 with anti-CCP is apparently due to strong linkage disequilibrium with nearby HLA genes. There is probably no major role of BTNL2 in the pathogenesis of RA, as hypothesized by Orozco et al. [16]. Our calculations also indicate that PTPN22 was not associated with either anti-CCP or RF (Table 4).

Table 3: Top-ranked genes associated with RA on chromosome 6

\begin{tabular}{lccc}
\hline Gene symbol & No. SNPs $^{\mathbf{a}}$ & SC $^{\mathbf{b}}$ & p-Value $^{\mathbf{c}}$ \\
\hline HLA-DRBI & $6 / 6$ & 1.00 & $4.89 \times 10^{-114}$ \\
HLA-DRA & $10 / 10$ & 0.90 & $1.18 \times 10^{-86}$ \\
BTNL2 & $10 / 10$ & 0.36 & $6.86 \times 10^{-81}$ \\
HLA-DQBI & $8 / 10$ & 0.36 & $1.86 \times 10^{-77}$ \\
HLA-DQA2 & $10 / 10$ & 1.00 & $6.19 \times 10^{-74}$ \\
HLA-DQAI & $5 / 5$ & 1.00 & $2.78 \times 10^{-72}$ \\
C6orfI0 & $10 / 10$ & 0.88 & $9.01 \times 10^{-70}$ \\
LOC40I252 & $9 / 10$ & 0.82 & $7.15 \times 10^{-51}$ \\
HLA-DOB & $10 / 10$ & 1.00 & $8.00 \times 10^{-32}$ \\
\hline
\end{tabular}

${ }^{a}$ The number of selected SNPs and the total number of SNPs that were genotyped.

bSC, stability coefficient.

'The adjusted $p$-values (no clustering) for all genes in the table are less than 0.001 ( 1,000 permutations). No clustering was performed.

\section{Discussion}

Our findings indicate that, in addition to genes on chromosome 6, PTPN22, TRAF1, ADAM15, AGPAT2, and a number of other genes shown in Table 2 may be associated with RA. MI clustering increased the overall ranks of PTPN22, AGPAT2, ADAM15, and TRAF1. In contrast, AS clustering lowered the rank of AGPAT2.

MI clustering seems to perform better than AS clustering in association studies because MI exhibited clusters of similar haplotypes [13] but AS produced clusters of ancestral populations [14]. The allelic test based on single SNPs outside of chromosome 6 showed that PTPN22, TRAF1, and ADAM15 were in the top 25 genes (non-adjusted $p$-values: $1.21 \times 10^{-10}, 8.37 \times 10^{-8}, 2.00 \times$ $10^{-7}$ respectively) except for AGPAT2 (non-adjusted $p$-value: $\left.1.69 \times 10^{-4}\right)$. As a result, AGPAT2 may have been overlooked in previous SNP-based analyses.

The genetic architecture of ADAM15 is noteworthy. The length of ADAM15 is one-third of the average human gene (the shortest of multiple-exon ADAM genes). It is comprised of 23 exons (2.5 times that of the human

Table 4: BTNL2 and PTPN22 loci associated with anti-CCP and RF

\begin{tabular}{|c|c|c|c|c|}
\hline \multirow[b]{2}{*}{ Trait } & \multicolumn{2}{|c|}{ BTNL2 gene } & \multicolumn{2}{|c|}{ PTPTN22 gene } \\
\hline & anti-CCP & $\mathbf{R F}$ & anti-CCP & RF \\
\hline $\begin{array}{l}\text { SNPs selected/ } \\
\text { total no. SNPs } \\
\text { genotyped }\end{array}$ & $6 / 6$ & $10 / 10$ & $6 / 7$ & $6 / 7$ \\
\hline SC & 1.00 & 1.00 & 1.00 & 0.88 \\
\hline$p$-Value & $5.33 \times 10^{-166}$ & $4.98 \times 10^{-1}$ & $5.40 \times 10^{-2}$ & $8.60 \times 10^{-1}$ \\
\hline Adjusted $p$-value & $<0.001$ & 0.560 & 0.026 & 0.870 \\
\hline
\end{tabular}


average) and 22 introns (the average length is less than one-tenth of the human average). At least 13 different splice variants have been found in normal human tissues [17]. These facts suggest that the identification of ADAM15 may be hindered due to its complex splicing.

The deterioration of arthritic joints is due to the fact that cells in synovial membranes that produce synovial fluids secrete several enzymes, collectively called matrix metalloproteinases (MMPs). MMPs are capable of degrading different types of extracellular matrix proteins, including bone and cartilage. A disintegrin and metalloproteinases $(A D A M s)$ is a new family of metalloproteinases that shares structural similarities with MMPs as well as snake venom metalloproteinases. A high level of ADAM15 mRNA expression has been found in RA synovium [18]. As RA progresses, the synovial tissue expands and forms destructive panni - flaps of tissue hanging on synovium and particularly on cartilages. The tissue hyperplasia induces oxygen deficiency, which necessitates more blood vessels to supply oxygen. An amplification loop begins and therefore promotes angiogenesis, the formation of new blood vessels arises from pre-existing blood vessels. Because angiogenesis is a crucial step to progress bone damage, a blockade of angiogenesis would prevent the delivery of oxygen and nutrients to the inflammatory site. Inhibiting vascular endothelial growth factor $(V E G F)$, which is a key factor in angiogenesis, showed a reduction in disease severity in animal models [19]. ADAM15 expression was up-regulated by VEGF, and linearly correlated with vascular density in synovial tissue [18]. A recent study suggested that VEGF and ADAM15 participate in an amplification loop. Inhibiting ADAM15 suppressed ocular neovascularization, a form of angiogenesis in eyes [20].

Although the mRNA expression level of ADAM15 was strongly associated with the progression and the severity of RA, the genetic predisposition to RA has not been elucidated yet. More association studies of ADAM15 in other independent populations, as well as better understanding of ADAM15 functions, are needed to confirm its effects in genetic predisposition to RA.

The involvement of AGPAT2 in RA is not straightforward. Generally, defects in AGPAT2 cause inherited lipodystrophy, a genetic disorder characterized by the selective loss of adipose tissue (fat loss) [21]. AGPAT2 is a crucial enzyme in biosynthesis of triacylglycerol, which is a primary form of fat stored in white fat cells. Thus, AGPAT2 is relevant to the formation of adipose tissue. White adipose tissue is ubiquitous in human joints. Although it used to be regarded simply as energy storage, it is now accepted that white adipose tissue is an endocrine organ that produces a variety of signaling proteins called adipokines. A significant level of three adipokines (leptin, adiponectin, resistin) has previously been found in synovial fluid of RA and osteoarthritis patients [22]. The adipokines play an important role in cross-talk between inflammatory system and adipocytes. It was concluded that the products of adipose tissue contribute to inflammatory and degenerative processes in common joint diseases [22]. Therefore, AGPAT2 may mediate inflammatory joints via adipose tissue.

The significant $p$-value of TRAF1 in Table 2 replicates the recent discovery of its association with predisposition to RA [5]. However, we could not replicate STAT4 and OLIG3/TNFAIP3's association with RA (non-adjusted $p$-value $=0.04$ and 0.02). Our failure to replicate STAT4 and OLIG3/TNFAIP3 may be due to the lack of a sufficiently large number of subjects as compared with the original study $[3,4,23]$. The other genes in Table 2 (C16orf57, KRTAP10-6, DEDD2, ODF3L1, RSHL1, and CDC42BPG) may contribute to RA, but no relevant literature suggests their potential role in the pathogenesis of RA.

We used the SNP annotation provided by Genetic Analysis Workshop 16 that assigns each SNP to one gene. There are 360,389 SNPs $(67.78 \%)$ that are in gene deserts and are farther than 10,000 bp from the closest genes. A reviewer's suggestion is to define intergenic units and test them separately. Defining intergenic units may identify more genomic regions contributing to RA, but the results for ADAM15 and AGPAT2 still hold, because they contain only SNPs nearby (distance $<5,000 \mathrm{bp}$ ).

\section{Conclusion}

RST may offer an effective alternative approach for SNP selection for genetic association studies. The stability coefficients can be used to filter out those genes that are statistically significant, but not stable. Consequently, this may help to increase our chance of distinguishing truly associated genes from those false signals. Our genebased analysis has led to the identifications of a number of novel genes, with ADAM15 and AGPAT2 having plausible biological mechanisms related to development of RA. These two genes may contribute a genetic predisposition to an abnormality in angiogenesis and adipose tissue, which are important factors in the progression of RA.

\section{List of abbreviations used}

anti-CCP: anti-cyclic citrullinated peptide; AS: Allele sharing; GDR: Generalized dynamic reduct; HLA: Human leukocyte antigen; MI: Mutual information; MMPs: Matrix metalloproteinases; NARAC: North American Rheumatoid Arthritis Consortium; RA: Rheumatoid 
arthritis; RF: Rheumatoid factor; RST: Rough set theory; SC: Stability coefficient; SNP: Single-nucleotide polymorphism; VEGF: Vascular endothelial growth factor.

\section{Competing interests}

The authors declare that they have no competing interests.

\section{Authors' contributions}

All authors participated in the design of the study. DHB and JYL performed data preprocessing. JSL and ZW performed statistical analysis. CA performed computer programming. HZ coordinated and helped to draft the manuscript. All authors read and approved the final manuscript.

\section{Acknowledgements}

The Genetic Analysis Workshops are supported by NIH grant ROI GM03I575 from the National Institute of General Medical Sciences. This work was supported in part by Yale MPSY Thai-U.S. Drug Dependence Genetics Research Training Program by Fogarty/NIH I D43 TW06I66, and NIH grants GM59507 and TI5 LM07056. We thank Alfonso Buil for his suggestion on the clustering algorithm used in this paper. We thank two reviewers for their helpful comments.

This article has been published as part of BMC Proceedings Volume 3 Supplement 7, 2009: Genetic Analysis Workshop 16. The full contents of the supplement are available online at http://www.biomedcentral.com/ $\underline{\mid 753-6561 / 3 \text { ? issue }=\text { S7. }}$.

\section{References}

I. Orozco G, Rueda B and Martin J: Genetic basis of rheumatoid arthritis. Biomed Pharmacother 2006, 60:656-662.

2. The Wellcome Trust Case Control Consortium: Genome-wide association study of 14,000 cases of seven common diseases and 3,000 shared controls. Nature 2007, 447:66I-678.

3. Plenge RM, Cotsapas C, Davies L, Price AL, de Bakker PI, Maller J, Pe'er I, Burtt NP, Blumenstiel B, DeFelice M, Parkin M, Barry R, Winslow W, Healy C, Graham RR, Neale BM, Izmailova E, Roubenoff R, Parker AN, Glass R, Karlson EW, Maher N, Hafler DA, Lee DM, Seldin MF, Remmers EF, Lee AT, Padyukov L, Alfredsson L, Coblyn J, Weinblatt ME, Gabriel SB, Purcell S, Klareskog L, Gregersen PK, Shadick NA, Daly MJ and Altshuler D: Two independent alleles at $6 \mathrm{q} 23$ associated with risk of rheumatoid arthritis. Nat Genet 2007, 39:|477-| 482.

4. Remmers EF, Plenge RM, Lee AT, Graham RR, Hom G, Behrens TW, de Bakker PI, Le JM, Lee HS, Batliwalla F, Li W, Masters SL, Booty MG, Carulli JP, Padyukov L, Alfredsson L, Klareskog L, Chen WV, Amos Cl, Criswell LA, Seldin MF, Kastner DL and Gregersen PK: STAT4 and the risk of rheumatoid arthritis and systemic lupus erythematosus. N Engl J Med 2007, 357:977-986.

5. Plenge RM, Seielstad M, Padyukov L, Lee AT, Remmers EF, Ding B, Liew A, Khalili H, Chandrasekaran A, Davies LR, Li W, Tan AK, Bonnard C, Ong RT, Thalamuthu A, Pettersson S, Liu C, Tian C, Chen WV, Carulli JP, Beckman EM, Altshuler D, Alfredsson L, Criswell LA, Amos Cl, Seldin MF, Kastner DL, Klareskog L and Gregersen PK: TRAFI-C5 as a risk locus for rheumatoid arthritis-a genomewide study. N Engl I Med 2007, 357:1 199-1209.

6. Neale BM and Sham PC: The future of association studies: gene-based analysis and replication. Am J Hum Genet 2004, 75:353-362.

7. Polkowski L: Rough Sets: Mathematical Foundations.Heidelberg, Physica-Verlag; 2002.

8. Wei L, Huang $C$ and Chen C: Data mining of the GAWI4 simulated data using rough set theory and tree-based methods. BMC Genet 2005, 6(SuppI I):SI33.
9. Bazan JG, Szczuka S and Wróblewski J: A new version of rough set exploration system. Rough Sets and Current Trends in Computing, LNCS 2475 Berlin, Springer: Alpigini JJ, Peters JF, Skowronek J, Zhong N 2002, 397-404.

10. Berkhin P: Survey of clustering data mining techniques. http:// citeseerx.ist.psu.edu/viewdoc/summary?doi=10.I.I.18.3739.

I I. Dunn J: Well separated clusters and optimal fuzzy partitions. J Cybernetics 1974, 4:95-104.

12. Kraskov A, Stögbauer H, Andrzejak RG and Grassberger P Hierarchical clustering based on mutual information. http:// arxiv.org/abs/q-bio/0311039.

13. Perera A, Buil A, Di Bernardo MC, Sabater-Lleal M, Pattini L, Souto JC, Fontcuberta J, Vallverdu M, Soria JM and Caminal P. Clustering of individuals given SNPs similarity based on normalized mutual information: F7 SNPs in the GAIT sample. Conf Proc IEEE Eng Med Biol Soc 2007, 2007:I23-I 26.

14. Gao $X$ and Starmer J: Human population structure detection via multi-locus genotype clustering. BMC Genet 2007 , 8: $|47|-2 \mid 56$

15. Irigoyen P, Lee AT, Wener MH, Li W, Kern M, Batliwalla F, Lum RF, Massarotti E, Weisman M, Bombardier C, Remmers EF, Kastner DL, Seldin MF, Criswell LA and Gregersen PK: Regulation of anticyclic citrullinated peptide antibodies in rheumatoid arthritis: contrasting effects of HLA-DR3 and the shared epitope alleles. Arthritis Rheum 2005, 52:3813-38I8.

16. Orozco G, Eerligh P, Sánchez E, Zhernakova S, Roep BO, GonzálezGay MA, López-Nevot MA, Callejas JL, Hidalgo C, Pascual-Salcedo D, Balsa A, González-Escribano MF, Koeleman BP and Martín J: Analysis of a function BTNL2 polymorphism in type I diabetes, rheumatoid arthritis, and systemic lupus erythematosus. Hum Immunol 2005, 66:|235-I24I.

17. Kleino I, Ortiz RM and Huovila AJ: ADAMI 5 gene structure and differential alternative exon use in human tissues. $B M C M O$ Biol 2007, 8:90.

18. Komiya K, Enomoto H, Inoki I, Okazaki S, Fujita Y, Ikeda E, Ohuchi E, Matsumoto H, Toyama $Y$ and Okada Y: Expression of ADAMI 5 in rheumatoid synovium: upregulation by vascular endothelial growth factor and possible implications for angiogenesis. Arthritis Res Ther 2005, 7:RI I58-RI I73.

19. Paleolog EM: Angiogenesis in rheumatoid arthritis. Arthritis Res 2002, 4(Suppl 3):S8I-S90.

20. Xie B, Shen J, Dong A, Swaim M, Hackett SF, Wyder L, Worpenberg S, Barbieri S and Campochiaro PA: An Adam I5 amplification loop promotes vascular endothelial growth factor-induced ocular neovascularization. J FASEB J 2008 22:2775-2783

21. Garg A: Acquired and inherited lipodystrophies. N Engl J Med 2004, 350:1220-1234

22. Toussirot E, Streit $G$ and Wendling D: The contribution of adipose tissue and adipokines to inflammation in joint diseases. Curr Med Chem 2007, I4:1095-1000.

23. Barton A, Thomson W, Ke X, Eyre S, Hinks A, Bowes J, Gibbons L Plant D, Wellcome Trust Case Control Consortium, Wilson AG, Marinou I, Morgan A, Emery P, YEAR consortium, Steer S, Hocking L, Reid DM, Wordsworth $P$, Harrison $P$ and Worthington J: Re-evaluation of putative rheumatoid arthritis susceptibility genes in the post-genome wide association study era and hypothesis of a key pathway underlying susceptibility. Hum Mol Genet 2008, 17:2274-2279.

24. Pawlak Z: Some issues on rough sets. Transactions on Rough Sets I, LNCS 3100 Berlin, Springer-Verlag: Peters JF, Skowron A, GrzymalaBusse JW, Kostek B, Swiniarski RW, Szczuka MS 2004, I-58. 